\title{
Reconhecimento da biblioteca universitária como um sistema adaptativo complexo: aplicação do framework gc@bu na Biblioteca Universitária da UFSC
}

\author{
Roberta Moraes de Bem \\ roberta.bem@ufsc.br \\ Biblioteca Central, Universidade Federal de Santa Catarina, Florianópolis, SC, Brasil \\ Joana Carla de Souza Matta Felicio \\ joana.felicio@ufsc.br \\ Biblioteca Central, Universidade Federal de Santa Catarina, Florianópolis, SC, Brasil
}

Maria Bernardete Martins Alves

m.alves@ufsc.br

Biblioteca Central, Universidade Federal de Santa Catarina, Florianópolis, SC, Brasil

Sigrid Karyn Weiss Dutra

sigrid.k.weiss@ufsc.br

Biblioteca Central, Universidade Federal de Santa Catarina, Florianópolis, SC, Brasil

Tatiana Rossi

tatiana.rossi@ufsc.br

Biblioteca Setorial, Centro de Ciências Agrárias. Universidade Federal de Santa Catarina,

Resumo: A Gestão do Conhecimento tem sido utilizada de forma estratégica nas organizações. Da mesma forma, as bibliotecas universitárias precisam utilizar-se desta ferramenta de gestão para obter vantagem competitiva e alavancar o uso do conhecimento. Com o apoio do Framework GC@BU a Biblioteca Universitária da UFSC iniciou as atividades de Gestão do Conhecimento em seu âmbito. Neste artigo apresenta-se o resultado do "Reconhecimento da Biblioteca Universitária", que consiste na primeira etapa de aplicação do GC@BU, em que esta é caracterizada como um Sistema Adaptativo Complexo. Com o apoio de uma Comissão criada para tal finalidade, realizaram-se discussões quinzenais acerca da temática, para identificar, avaliar e registrar os elementos fundamentais que constituem a biblioteca. Constata-se que a aplicação do GC@BU, ainda que de forma inicial, apresenta resultados positivos, verificando o quão específico, adaptativa e dinâmica é a ferramenta.

Palavras-chave: gestão do conhecimento; bibliotecas; teoria da complexidade.

Recognition of university library as a complex adaptive system: application of the framework gc@bu in the UFSC University Library

Abstract: Knowledge Management (KM) has been used strategically in organizations. Similarly, the university libraries must use this management tool to get competitive advantage and encourage the use of knowledge. Using the Framework GC@BU the UFSC University Library started the KM activities in its environment. This paper presents the results of "Recognition of University Library", which consists of the first application step of GC@BU, wherein it is characterized as a Complex Adaptive System (CAS). Supported by a committee created for this purpose, fortnightly discussions were held about the topic to identify, assess and record the main elements that make up the library. We check that the application of 
GC@BU, even if initially, showed positive results, checking how specific is the tool, adaptive and dynamic.

Keywords: knowledge management; libraries; complexity theory.

Reconocimiento de la biblioteca universitaria como un sistema adaptativo complejo (sac): aplicación del framework gc@bu en la Biblioteca Universitaria de la UFSC

Resumen: La Gestión del Conocimiento ha sido utilizada de forma estratégica en las organizaciones. De la misma forma, las bibliotecas universitarias necesitan utilizar esta herramienta de gestión para obtener ventaja competitiva e impulsar el uso del conocimiento. Con el apoyo del Framework GC@BU, la Biblioteca Universitaria de la UFSC inició las actividades de Gestión del Conocimiento en su ámbito. En este artículo se presenta el resultado del "Reconocimiento de la Biblioteca Universitaria", que consiste en la primera etapa de aplicación del GC@BU, en que esta es caracterizada como un Sistema Adaptativo Complejo. Con el apoyo de una Comisión creada para tal finalidad, se realizaron discusiones quincenales acerca de la temática, para identificar, evaluar y registrar los elementos fundamentales que constituyen la biblioteca. Se constata que la aplicación del GC@BU, aunque de forma inicial, presenta resultados positivos, verificando cuan específica, adaptativa y dinámica es la herramienta.

Palabras clave: gestión del conocimiento; bibliotecas; teoría de la complejidad. 


\section{Contextualização}

$\mathrm{Na}$ era do conhecimento as organizações são afetadas por mudanças expressivas, isto inclui as instituições educacionais e, consequentemente as suas bibliotecas, que são por natureza, fontes de organização, difusão e acesso ao conhecimento, responsáveis por dinamizar as atividades de ensino, oferecendo os recursos essenciais para a aprendizagem, 0 ensino e a pesquisa (LEMOS; MACEDO, 2004).

Tudo o que afeta as instituições educacionais impacta diretamente nas bibliotecas, e vice-versa, ou seja, a forma como a biblioteca desenvolve seus serviços e produtos também interfere diretamente no desempenho institucional. Ela é parte de um todo, seu acervo, serviços e produtos devem ser considerados como recursos pedagógicos essenciais.

Hoje as bibliotecas precisam ampliar seu papel e oferecer vantagem competitiva à instituição de ensino em que está inserida, ampliando seu papel e responsabilidades, o que inclui a gestão da informação e do conhecimento produzido na instituição. Isto significa que elas precisam criar e aprimorar a sua base de conhecimentos, seus ativos intelectuais, mediante à adoção de práticas de Gestão do Conhecimento (GC), valorizando os conhecimentos tácitos e explícitos, as experiências acumuladas de sua equipe proporcionando em algum nível a melhoria e a inovação. (XIN, 2011).

A base de todo o processo de atualização e inovação é o conhecimento que por sua vez é construído pelas pessoas que atuam na instituição de forma direta ou indireta. Os ativos intangíveis, ou capital intelectual, representam uma parcela significativa no valor total de uma organização (DAVENPORT; PRUSAK, 2003; NONAKA; TAKEUCHI, 1997), porém o Capital Intelectual $(\mathrm{Cl})$ não se limita pura e simplesmente ao conhecimento, mas também a capacidade que uma organização possui de aprender e de se adaptar às mudanças que ocorrem no mercado (MONAGAS-DOCASAL, 2012).

Pensando nesta necessidade de adaptação e de manter-se em um mercado, cada vez mais competitivo, a Gestão do Conhecimento surge como estratégia para alavancar os recursos existentes e potencializar a criação e utilização de novos conhecimentos. Para tanto, ferramentas e modelos de gestão estão disponíveis para apoiar estas iniciativas. O Framework de Gestão do Conhecimento para Bibliotecas Universitárias (GC@BU) apresentado na sequencia foi adotado para a implementação da GC na Biblioteca Universitária (BU) da Universidade Federal de Santa Catarina (UFSC). 


\section{Framework GC@BU}

O Framework GC@BU (Figura 1) é resultado de uma tese de doutorado (BEM, 2015) que propõe a implementação da GC com foco em bibliotecas universitárias. Dentre as suas características estão a adaptabilidade e o potencial para a aplicação. Foi construído a partir de revisão de literatura e submetido à avaliação de um grupo de gestores de bibliotecas universitárias, na ocasião do I Workshop Gestão do Conhecimento em Bibliotecas Universitárias $(2014)^{1}$.

Figura 1 - Framework de Gestão do Conhecimento para Bibliotecas Universitárias (GC@BU)

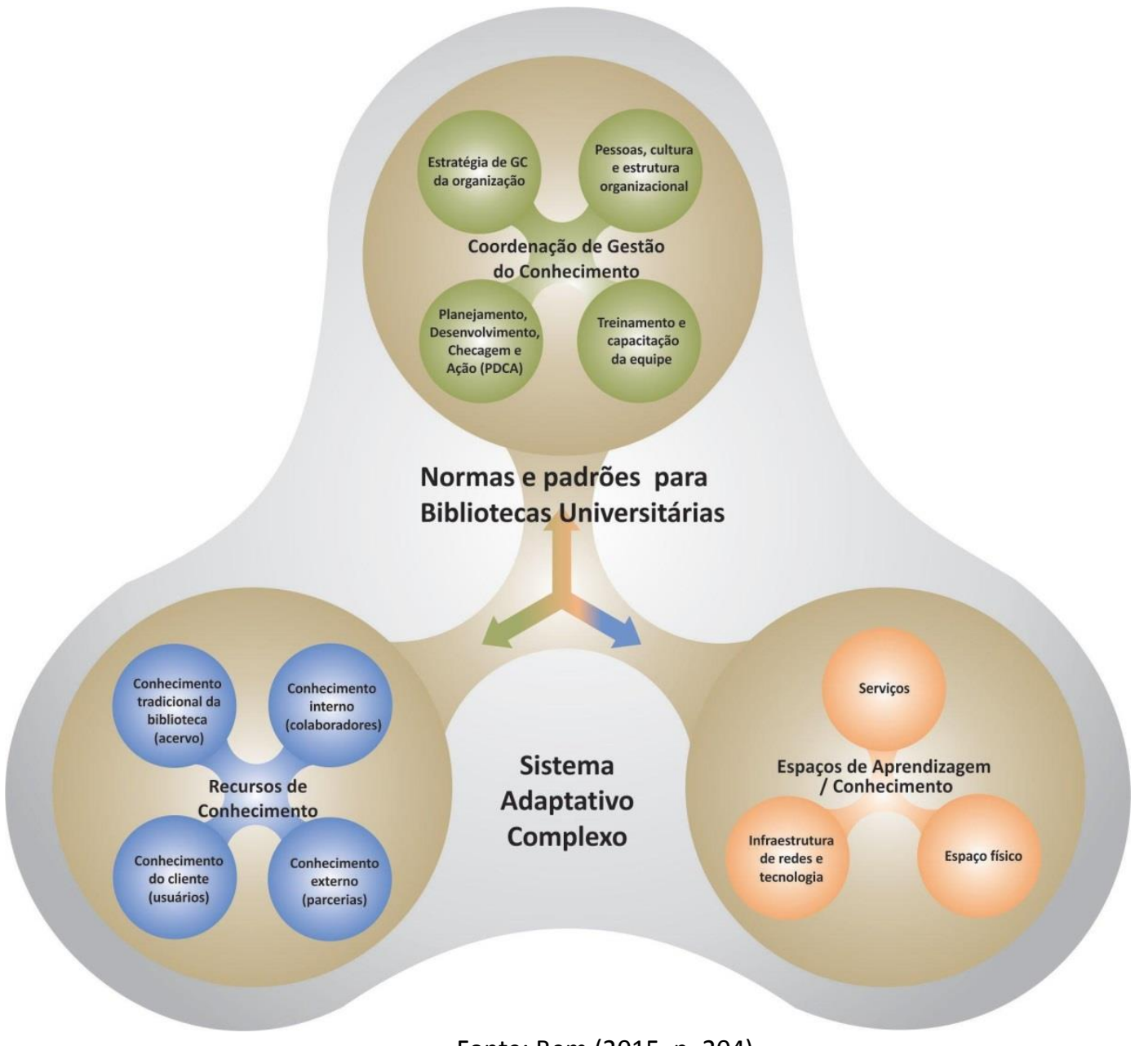

Fonte: Bem (2015, p. 204).

O GC@BU é composto de três módulos (BEM, 2015):

\footnotetext{
${ }^{1}$ Realizado na UFSC com o objetivo de aprofundar conhecimentos na área, além de avaliar o framework proposto. Disponível em: <http://workshopgestaoconhecimentobu.paginas.ufsc.br/>.
} 
a) Coordenação de Gestão do Conhecimento - este modulo baseia-se nas abordagens de Porumbeanu (2009), Castro (2005), Shuhuai et al. (2009), Pacheco et al. (2005) e nos princípios da eficácia institucional e da gestão/administração da Association of College and Research Libraries (ACRL, 2011), juntamente com seus respectivos indicadores. De acordo com Porumbeanu (2009), a coordenação de GC vai lidar com tudo o que compõe a estratégia de conhecimento da organização. De forma geral, é responsável pelo bom funcionamento do processo de GC no âmbito da biblioteca ou serviço de informação. Irá coordenar a elaboração e implementação de programas e sistemas de GC, encontrar novas fontes de conhecimento, identificar novas formas de uso efetivo do conhecimento na organização, entre outras funções. Os elementos que o compõem são:

- Estratégia de Gestão do Conhecimento da organização;

- Pessoas, cultura e estrutura organizacional;

- Treinamento e capacitação da equipe;

- Planejamento, Desenvolvimento, Checagem e Ação (PDCA).

b) Recursos de Conhecimento - é o módulo do Framework GC@BU que representa a essência das bibliotecas universitárias, considerando que trabalha o seu objetivo primordial, que é o de prover informações e conhecimentos aos seus usuários nos mais diferentes formatos e por meio dos mais diversos serviços, baseia-se nos seguinte autores: Association of College and Research Libraries (ACRL, 2011), Dong (2008), Brasil (2012), Corral e Sriborisutsakul (2010), Castro (2005), Porumbeanu (2009), Daneshgar e Parirokh (2012) e Merrick (2009). Os elementos que o compõem são:

- Conhecimento tradicional (acervo);

- Conhecimento interno (colaboradores);

- Conhecimento do cliente (usuários);

- Conhecimento externo (parcerias).

c) Espaços de Aprendizagem/Conhecimento - baseia-se nos princípios dos "learning commons", uma evolução do termo "information commons", que vem sendo utilizado para designar os espaços desenvolvidos com a finalidade de produção de conhecimento e aprendizagem - característica marcante das bibliotecas modernas - no sentido de dar apoio a um modelo de aprendizagem mais interativo. Os "learning commons" estão sendo estudados no contexto das bibliotecas universitárias, como espaços que integram uma série de elementos, considerando 
aspectos físicos e virtuais, associados à infraestrutura de redes e tecnologia, além de serviços e recursos humanos qualificados. Este módulo foi desenvolvido com base em três autores (ASSOCIATION OF COLLEGE AND RESEARCH LIBRARIES, 2011; SHUHUAl et al., 2009; CASTRO, 2005), sendo composto por três elementos:

- Serviços;

- Espaços;

- Infraestrutura de redes e tecnologias.

Conforme observado na Figura 1 o GC@BU possui um fundo em cinza e os módulos estão emoldurados por um tom dourado, eles representam a visão dos Sistemas Adaptativos Complexos (SAC) e as normas para bibliotecas universitárias da Association of College and Research Libraries, respectivamente.

A caracterização da biblioteca universitária como um SAC consiste na parte inicial da implementação, considerando que, apesar de tratar-se de um pressuposto teórico (BU como SAC), ela possui elementos próprios com suas respectivas planilhas de verificação. Essa representação será apresentada a seguir.

\subsection{A visão do Sistema Adaptativo Complexo na Biblioteca Universitária: GC@BU}

Essa forma de conceber a biblioteca universitária (como um SAC) baseia-se principalmente nas perguntas de Axelrod e Cohen (1999) - um plano para o usuário que pretende administrar a complexidade em seu sistema, ajudando-o a guiar-se no domínio da complexidade de um sistema particular. A Figura 2 apresenta os elementos que estão contidos nesta forma de caracterizar a biblioteca universitária, apresentados nas seções subsequentes. 
Figura 2 - Caracterização da Biblioteca Universitária como um Sistema Adaptativo Complexo

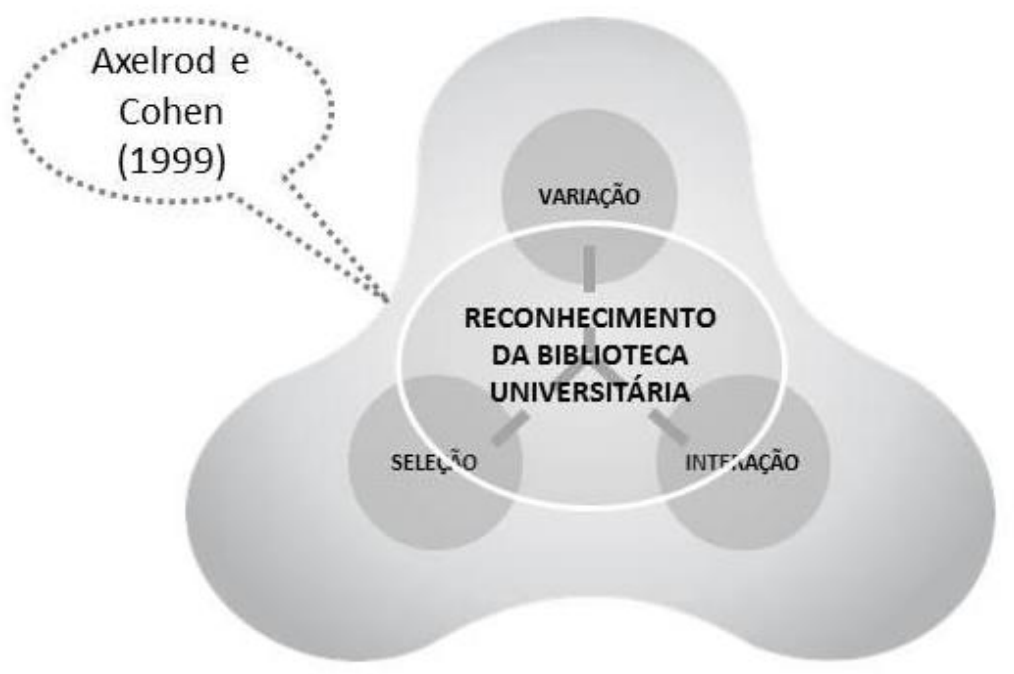

Fonte: Bem (2015, p. 206).

\subsubsection{Reconhecimento}

O principal elemento considerado nas bibliotecas universitárias sob a perspectiva dos SAC são os agentes, pois tudo gira em torno deles, especialmente em um contexto em que as relações humanas e as atividades de orientação e interação com os usuários estão em constante crescimento (BEM, 2015).

Entende-se por agentes, de acordo com Axelrod e Cohen (1999): elementos que têm a habilidade de interagir com o seu meio, incluindo outros agentes. Os agentes são caracterizados por três atributos: localização (onde o agente opera), capacidades (como o agente pode afetar o mundo) e memória (que impressões o agente pode trazer do seu passado).

"Agente não é apenas o conceito central da teoria da complexidade, mas o componente mais elementar na implantação de sistemas de GC" (LI; ZHANG, 2011, p. 53).

É importante que a biblioteca universitária conheça seus agentes incluindo todos os seus atributos, pois eles correspondem ao principal foco da GC, além dos demais elementos que estão atrelados a eles, como as estratégias que utilizam, os processos organizacionais em que atuam, o conhecimento sobre a organização, entre outros (BEM, 2015). 


\subsubsection{Variação}

O elemento variação tem sua importância caracterizada pelo atributo de servir de matéria-prima para a adaptação, já que se caracterizam justamente por processos de mudança. (AXELROD; COHEN, 1999).

No contexto da biblioteca universitária é importante que se tenha consciência das modificações que têm sido implantadas e o porquê delas, incluindo fatores e agentes que possam estar envolvidos (BEM, 2015).

\subsubsection{Interação}

A interação caracteriza a vivacidade do SAC. Padrões de interação ajudam a determinar o que será bem-sucedido para os agentes de um sistema, e isso, em troca, ajuda na dinâmica dos padrões de interação entre eles. Os padrões de interação precisam ser conhecidos e monitorados, porque da mesma forma que as interações podem espalhar eventos positivos para o sistema, o contrário também é verdadeiro.

\subsubsection{Seleção}

A seleção é resultado de um processo, como o aprendizado, por exemplo. Nem sempre a seleção é benéfica e pode resultar em mudanças nos agentes, nas estratégias e populações. Por isso, é importante observar: os critérios de sucesso; determinar se a seleção está no nível dos agentes ou das estratégias; atribuir crédito para o sucesso e o fracasso; criar novos agentes ou estratégias (COELHO, 2001).

Quando um processo de seleção leva ao sucesso, então o chamamos de adaptação (AXELROD; COHEN, 1999). E é com base na adaptação que a questão da seleção é vista no GC@BU, buscando sempre a melhoria contínua. Na seção 3 será apresentado um exemplo de aplicação da visão dos SAC em uma biblioteca universitária, a BU/UFSC.

\section{Reconhecimento da BU/UFSC}

A BU/UFSC é uma unidade organizacional vinculada à Reitoria. Com aproximadamente 50 anos de existência, a BU/UFSC tem como missão "participar no processo de disseminação da informação e do conhecimento de forma articulada para o desenvolvimento das atividades de ensino, pesquisa, extensão e à administração da UFSC" (UNIVERSIDADE FEDERAL DE SANTA CATARINA, 2015b).

A estrutura organizacional da BU/UFSC é constituída de uma direção, responsável pela coordenação geral do sistema de bibliotecas que se compõe de: Biblioteca Central (BC); 
Biblioteca Setorial do Centro de Ciências da Saúde - Medicina (BSCCSM); Biblioteca Setorial do Centro de Ciências Físicas e Matemática (BSCFM); Biblioteca Setorial do Colégio de Aplicação (BSCA); Biblioteca Setorial do Centro de Ciências Agrárias (BSCCA); Biblioteca Setorial do Centro de Ciências da Educação (BSCED); Biblioteca Setorial do Campus de Araranguá (BSARA); Biblioteca Setorial do Campus de Curitibanos (BSCUR); Biblioteca Setorial do Campus de Blumenau (BSBLU) e Biblioteca Setorial do Campus de Joinville (BSJOI). Estão também sob a coordenação da direção da BU/UFSC a Divisão de Desenvolvimento de Coleções e Tratamento da Informação (DECTI), a Divisão de Automação e Informática (DAINF), o Repositório Institucional da UFSC e o Portal de Periódicos da UFSC (UNIVERSIDADE FEDERAL DE SANTA CATARINA, 2015b).

Conforme mencionado anteriormente, o Framework GC@BU é resultado de uma tese de doutorado. A autora, que integra à equipe da BU/UFSC, foi convidada à realizar a aplicação desta proposta na biblioteca, conforme a política da BU de valorizar o conhecimento desenvolvido pelos seus servidores como ferramenta de melhoria e inovação. Em maio de 2015 foi nomeada, por meio da Portaria no 849/2015/GR, de 25 de maio de 2015 uma "comissão permanente para implantar práticas e projetos de gestão do conhecimento apoiados no Framework GC@BU, com o objetivo de melhorar o fluxo e mapeamento de informações e conhecimentos no Sistema de Bibliotecas da UFSC" (UNIVERSIDADE FEDERAL DE SANTA CATARINA, 2015a, p. 1). Desde então, essa Comissão passou a reunir-se com frequência quinzenal para implantar as práticas de GC na BU/UFSC.

A etapa inicial de implementação do Framework GC@BU foi a caracterização da biblioteca universitária como um SAC, que tem como ponto de partida o seu reconhecimento. Trata-se de um exercício de identificar, avaliar e registrar os elementos fundamentais que constituem a biblioteca, tal como responder as questões: Quem somos? Onde estamos? O que fazemos? Como fazemos? Por que fazemos? Para quem fazemos? O reconhecimento da biblioteca universitária é uma forma de realizar um diagnóstico com vistas a ações futuras.

Utilizando-se as planilhas de verificação disponíveis no Framework GC@BU (ver Anexo 1), que apresentam os critérios de verificação a serem analisados, a Comissão de GC@BU/UFSC iniciou os trabalhos de reconhecimento da BU/UFSC, conforme descrito nas subseções a seguir.

Após o preenchimento das planilhas de verificação do Framework GC@BU elaborou-se um mapa conceitual com a finalidade de organizar e representar visualmente os itens que foram verificados e analisados, como demonstrado na Figura 3.

Para esta atividade utilizou-se o software Cmap Tools, uma ferramenta para elaboração de mapa conceitual disponível gratuitamente na Internet. A Comissão avaliou outras RICI: R.Ibero-amer. Ci. Inf., ISSN 1983-5213, Brasília, v. 9, n. 1, p. 87-107, jan./jun.2016. 
ferramentas semelhantes e concluiu que o Cmap Tools atenderia a finalidade da atividade. Utilizou-se variadas formas e cores para representar os diferentes conceitos, e de frases de ligação para especificar as relações entre os conceitos apresentados.

Também foram incluídas notas para explicar melhor um determinado conceito ou exemplificá-los, além de links para ferramentas e documentos disponibilizados na web, conforme menção no mapa.

Figura 3 - Mapa conceitual: Reconhecimento da BU/UFSC

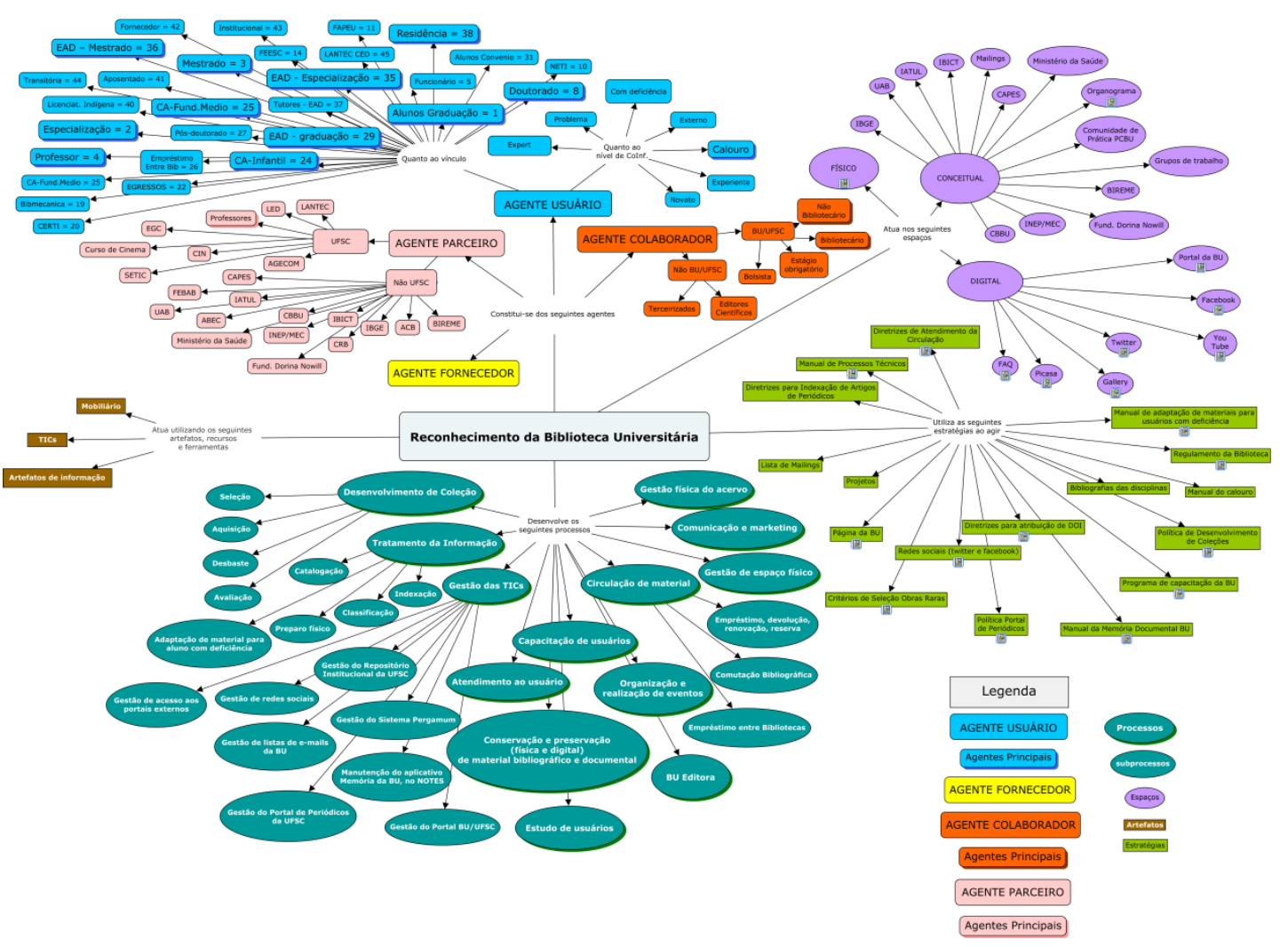

Fonte: Comissão de GC da BU/UFSC. Implementação do Framework GC@BU (2015).

\subsection{Agentes}

O primeiro critério de verificação diz respeito a "identificação e categorização dos agentes em tipologias e populações" (BEM, 2015, p. 209-211).

A Comissão de GC@BU/UFSC identificou quatro principais agentes: usuário, colaborador, fornecedor e parceiro, os quais estão apresentados no mapa conceitual, figura 3.

Como agente usuário, foram considerados todos os usuários atendidos na BU/UFSC como: alunos de graduação e pós-graduação, egressos, servidores, usuários de convênios, dentre outros. Esta identificação foi feita com base no catálogo automatizado da biblioteca, 
denominado Sistema Pergamum, no qual os usuários estão divididos em 28 categorias distintas, como pode ser observado pela Figura 3.

No agente colaborador foram incluídos todos os funcionários, efetivos ou não, que trabalham na biblioteca, dividindo-os em "BU/UFSC", aqueles que são colaboradores internos à BU e os "Não BU/UFSC" que são os funcionários terceirizados e demais colaboradores externos.

Os fornecedores de livros, bases de dados, materiais de expediente, dentre outros, foram representados como agente fornecedor.

Como agente parceiro incluiu-se as pessoas ou populações que contribuem com ou para os serviços da biblioteca de alguma forma. O agente parceiro foi subdividido em: "UFSC", aqueles que fazem parte da comunidade universitária, como os professores, os departamentos que prestam serviços para a BU/UFSC ou a auxilia de alguma forma, entre outros e, os denominados "Não UFSC", que seriam os parceiros externos com quem a BU/UFSC mantem algum tipo de relacionamento, como as Associações, Conselhos, Federações, Institutos, etc.

O segundo critério de verificação, ainda relacionado aos agentes, visa "conhecer os principais agentes a serem focados pela BU" (BEM, 2015, p. 209-211). Assim, entre as categorias e subcategorias de agentes identificadas no critério anterior, considerou-se como principais focos de atuação da BU/UFSC: a) agente usuário - identificaram-se os alunos de graduação e pós-graduação presenciais e a distância, os alunos residentes, os alunos do Colégio de Aplicação (educação infantil, ensino fundamental e médio), os calouros e os professores; b) agente colaborador - destacaram-se os bibliotecários e não bibliotecários, categorizados como "BU/UFSC"; c) agente parceiro - o foco é no professor da UFSC.

Ainda no que se refere a categorização dos agentes, o quinto critério de verificação diz respeito ao "conhecimento das classificações que os próprios agentes fazem para outros agentes em populações e tipologias" (BEM, 2015, p. 209-211). Neste critério a Comissão de GC@BU/UFSC criou categorias de agentes (Quadro 1) com base no nível de competência informacional. Para ajudar na definição da nomenclatura, recorreu-se, também, à literatura da área.

Quadro 1 - Categorias de agentes

\begin{tabular}{|c|l|}
\hline \multicolumn{1}{|c|}{ TIPO DE USUÁRIO } & \multicolumn{1}{c|}{ DESCRIÇÃO } \\
\hline usuário problema & $\begin{array}{l}\text { pessoa que tem comportamento incompatível com as normas de } \\
\text { funcionamento do centro de informação (CUNHA; CAVALCANTI, } \\
\text { 2008); }\end{array}$ \\
\hline
\end{tabular}




\begin{tabular}{|l|l|}
\hline usuário calouro & $\begin{array}{l}\text { pessoa recém chegada na instituição com demandas básicas como } \\
\text { informações de empréstimo, cadastro; }\end{array}$ \\
\hline usuário novato & $\begin{array}{l}\text { pessoa que nunca utilizou a unidade de informação (CUNHA; } \\
\text { CAVALCANTI, 2008); }\end{array}$ \\
\hline usuário expert & $\begin{array}{l}\text { pessoa com dúvidas pontuais e difíceis de serem respondidas, } \\
\text { exigindo um profissional extremamente qualificado e dispendendo } \\
\text { bastante tempo deste; }\end{array}$ \\
\hline usuário externo & $\begin{array}{l}\text { pessoa que embora não pertencendo a determinada instituição, tem } \\
\text { autorização para utilizar os produtos e serviços da biblioteca } \\
\text { (CUNHA; CAVALCANTI, 2008); }\end{array}$ \\
\hline usuário experiente & $\begin{array}{l}\text { pessoa que utiliza de forma regular a unidade de informação e está } \\
\text { familiarizado com a maioria de suas funções (CUNHA; CAVALCANTI, } \\
\text { 2008); }\end{array}$ \\
\hline usuário com deficiência & $\begin{array}{l}\text { pessoa com deficiência física e ou de aprendizagem que demandam } \\
\text { produtos de informação adaptados e atendimento especializado. }\end{array}$ \\
\hline
\end{tabular}

Fonte: Comissão de GC@BU/UFSC (2015); Cunha e Cavalcanti (2008).

\subsection{Estratégias}

O terceiro critério de verificação na etapa de reconhecimento da biblioteca, diz respeito a "identificação das principais estratégias, além de ideias, regras, rotinas e normas utilizadas pelos agentes ao agirem" (BEM, 2015, p. 209-211).

No caso da BU/UFSC, a Comissão identificou que as principais estratégias utilizadas são os manuais, diretrizes, políticas, regulamentos, mailings, projetos, bibliografias das disciplinas, página da BU na Web e redes sociais, como demonstrado na Figura 3. Porém, a Comissão verificou ainda que, embora tenha identificado as estratégias, algumas não existem formalmente, sendo necessário a previsão de uma ação para corrigir este fato.

\subsection{Processos}

O quarto critério de verificação consiste em identificar se a BU/UFSC possui seus processos de trabalho mapeados. De acordo com este critério:

Caso a BU ainda não tenha o mapeamento de processos (conjunto de atividades realizadas para atingir um serviço) estabelecido de modo formal, será necessário desenvolvê-lo. A partir do mapeamento dos processos-chave da BU é possível identificar, entre outros elementos, os pontos fortes e fracos, os conhecimentos e agentes envolvidos, o nível de complexidade da operação (BEM, 2015, p. 209-211).

A Comissão identificou alguns processos e subprocessos, entretanto, foi solicitado apoio aos demais integrantes da equipe da BU/UFSC. Os processos e subprocessos estão 
representados na Figura 3, sendo os processos: Desenvolvimento de Coleção; Tratamento da Informação; Conservação e preservação de material bibliográfico e documental; Atendimento ao usuário; Estudo de usuários; Capacitação de usuários; Circulação de material bibliográfico e documental; Comunicação e marketing; BU Editora; Gestão física do acervo; Gestão de espaço físico; Gestão das Tecnologias de Informação e Comunicação; Organização e realização de eventos.

Constatou-se que a BU/UFSC necessita conhecer e formalizar os seus processos, tendo em vista que a maioria deles não estão mapeados de maneira formal, havendo apenas alguns fluxogramas, manuais, desenvolvidos pela equipe de forma isolada. Há a necessidade de que sejam mapeados graficamente, podendo ser facilmente compreendidos de forma visual.

\subsection{Artefatos}

Para execução das estratégias por parte dos agentes, são utilizados artefatos, recursos e ferramentas (BEM, 2015). Neste critério identificou-se: o mobiliário de forma geral; os artefatos de informação como livros, periódicos, artigos, bases de dados, dentre outros; e as tecnologias de informação e comunicação, tais como: os computadores e similares, ferramentas da Internet e computacionais, softwares variados, catálogos colaborativos, dentre outros. Este critério, assim como os demais, também está representado (Figura 3).

\subsection{Espaços}

Este critério diz respeito aos "espaços físico, conceitual e digital em que os agentes atuam" (BEM, 2015, p. 209-211).

Para descrição do espaço físico são incluídas informações referentes a localização geográfica e demais informações físicas e de infraestrutura, tais como: dimensão dos ambientes, informações referentes a climatização, iluminação, mobiliários, capacidade para alocar pessoas, plantas, fotos, vídeos, dentre outras (BEM, 2015). Na BU/UFSC observou-se que não há um levantamento padronizado destas informações nas bibliotecas, entretanto, na instituição já foi desenvolvido um sistema para esta finalidade. Trata-se do Sistema Integrado de Espaço Físico (SIEF) que possibilitará cadastrar os ambientes disponíveis nas bibliotecas da UFSC, sendo possível descrever os ambientes, incluindo informações relacionadas a dimensões; acessibilidade (arquitetura, mobiliário); conforto (climatização, iluminação, odor, ruído, mobiliários) e equipamentos (mobiliários disponíveis, computadores, arescondicionados, ventiladores, projetor multimídia, microfones, etc.). O SIEF ainda possibilita incluir fotos para ilustrar os ambientes cadastrados, além de fazer a gestão de patrimônio 
integrada. Este item ainda está em processo de descrição e avaliação pela Comissão de GC@BU/UFSC.

O espaço conceitual refere-se a uma determinada organização que leva em consideração assuntos, temáticas, estruturas, agrupamentos (BEM, 2015). Na BU/UFSC identificou-se como espaço conceitual: o organograma, as comunidades de prática, mailings, grupos de trabalho, parcerias institucionais, entre outros.

O espaço digital, por sua vez, suporta conteúdos em formatos eletrônicos, que podem ou não serem virtuais (BEM, 2015). Foram identificados como espaço digital na BU/UFSC: o Portal da BU (página na Internet), as Frequently Asked Questions (FAQ) e as redes sociais (Figura 3).

\section{Considerações Finais}

Considerando o objetivo da BU/UFSC em manter-se competitiva e aliada aos benefícios que a GC pode proporcionar, a adoção de um framework traz a sistemática necessária para a o planejamento, desenvolvimento e avaliação das iniciativas de GC. Neste sentido, a experiência de Reconhecimento da BU/UFSC, por meio dos encontros quinzenais com a comissão proporcionou um resultado além do esperado. Na medida em que conhecimentos compartilhados e trabalhados por uma equipe de quinze pessoas, para tornaram-se de entendimento coletivo, necessitam de um trabalho de mediação e organização, para que as informações não se perdessem e pudessem ser registradas, da forma como a ferramenta GC@BU propunha.

Um dos critérios de sucesso é justamente a composição da equipe de modo voluntário, não caracterizando uma demanda top-down com enfoque formal. Embora, sua formalização tenha acontecido, por meio de uma portaria, a motivação para o trabalho se deu de modo espontâneo, constituindo uma equipe motivada, comprometida e produtiva.

Ademais, o detalhamento das informações coletadas para o cumprimento dos critérios de verificação da planilha de reconhecimento, impôs a sua representação de forma gráfica. Considerando que até o momento foi implantado apenas um dos elementos do plano de fundo do GC@BU (visão dos SAC) constatou-se o quão específico, adaptativo e dinâmico é o GC@BU, de modo que as demandas que surgiram a partir desta atividade inicial (formalização e mapeamento de processos, criação de grupos de trabalho, gestão de espaços físicos, entre outras) tornam-se pequenos "braços" dentro de uma imprevisível rede de relacionamentos, assim como são as bibliotecas universitárias no contexto mutável em que atuam. 


\section{Agradecimentos}

Agradecimentos à equipe que integra a Comissão de GC@BU/UFSC pelas contribuições nas discussões realizadas em reuniões periódicas, na identificação, registro e avaliação dos critérios de verificação do Framework GC@BU com vistas ao Reconhecimento da BU/UFSC como um SAC. Agradecimentos extensivos, também, à toda a equipe BU/UFSC.

Christianne Coelho de Souza Reinisch Coelho, Gabriel Araldi Walter, Gertrudes Aparecida Dandolini, Hilda Carolina Feijó, João Artur de Souza, Júlia Miranda Bressane, Leila Cristina Weiss, Leonardo Ripoll Tavares Leite, Márcia Dietrich Santiago, Márcia Prim, Maria Gorete Monteguti Savi, Ricardo Krüger Tavares, Tânia Regina Pereira Lopes e Wanessa C. da Silva.

\section{Referências}

ASSOCIATION OF COLLEGE \& RESEARCH LIBRARIES. Standards for Libraries in Higher Education. Chicago, 2011.2 Disponível em: <http://www.ala.org/acrl/sites/ala.org.acrl/files/content/standards/slhe.pdf>. Acesso em: 9 jul. 2015.

AXELROD, R. M.; COHEN, M. D. Harnessing complexity: organizational implications of a scientific frontier. New York: Free Press, 1999.

BEM, R. M. de. Framework de Gestão do Conhecimento para bibliotecas universitárias. 2015. $344 p$. Tese (Doutorado em Engenharia e Gestão do Conhecimento) -Universidade Federal de Santa Catarina, Florianópolis, 2015. Disponível em: <http://tede.ufsc.br/teses/PEGC0364T.pdf>. Acesso em: 20 jul. 2015.

BRASIL. Ministério da Educação. Instrumento de avaliação dos cursos de graduação presencial e a distância. Brasília, 2012. Disponível em: <http://download.inep.gov.br/educacao_superior/avaliacao_cursos_graduacao/instrumentos/ 2012/instrumento_com_alteracoes_maio_12.pdf>. Acesso em: 4 ago. 2015.

CASTRO, G. de. Gestão do conhecimento em bibliotecas universitárias: um instrumento de diagnóstico. 2005. 160 p. Dissertação (Mestrado em Ciência da Informação) -Universidade Federal de Santa Catarina, Florianópolis, 2005. Disponível em: <http://pgcin.paginas.ufsc.br/files/2010/10/CASTRO-Gardenia.pdf>. Acesso em: 26 jul. 2015.

COELHO, C. de S. R. Complexidade e sustentabilidade nas organizações. 2001.189 p. Tese (Doutorado em Engenharia de Produção) - Universidade Federal de Santa Catarina, Florianópolis, 2001. Disponível em: <http://www.tede.ufsc.br/teses/PEPS1753T.pdf>. Acesso em: 26 julho 2015.

CORRALL, S.; SRIBORISUTSAKUL, S. Evaluating intellectual assets in university libraries: a multisite case study from Thailand. Journal of Information \& Knowledge Management, v. 9, n. 3, p.277-290, 2010. Disponível em: <http://dx.doi.org/10.1142/S021964921000267X>. Acesso em: 26 jul. 2015.

CUNHA, Murilo Bastos da; CAVALCANTI, Cordélia R. Dicionário de biblioteconomia e arquivologia. Brasília: Briquet De Lemos, 2008. p. 373. 
DANESHGAR, F.; PARIROKH, M. An integrated customer knowledge management framework for academic libraries. The Library Quarterly, v. 82, n. 1, p. 7-28, jan. 2012. Disponível em: $<$ http://search.ebscohost.com/login.aspx?direct=true\&db=lih\&AN=69861166\&lang=ptbr\&site=ehost-live $>$. Acesso em: 26 jul. 2015.

DAVENPORT, T.; PRUSAK, L. Working knowledge: how organizations manage what they know. Boston: Harvard Business School Press, 1998. In: EVANGELISTA, Amândio; DONATO, Antonio; CARVALHO, Fernando; NEVES, Humberto. A gestão do conhecimento nas organizações: comportamento organizacional. Portugal: Instituto Superior de Economia e Gestão, 2003. DONG, E. X. Using blogs for knowledge management in libraries. CALA Ocasional Paper Series. n. $2, \quad$ p. $1-7, \quad$ nov. 2008.2 Disponível em: $<$ http://search.ebscohost.com/login.aspx?direct=true\&db=lih\&AN=35849323\&lang=ptbr\&site=ehost-live $>$. Acesso em: 26 jul. 2015.

LEMOS, L. O. C.; MACEDO, C. A cooperação entre bibliotecas do ensino superior: passado, presente e perspectiva futura. JORNADAS PORBASE: novos contextos para a cooperação, 9. Lisboa. 2004. Anais eletrônicos ... $<$ http://sigarra.up.pt/up/web_gessi_docs.download_file?p_name=F1606244585/Comunicacao .pdf>. Acesso em: 22 jun. 2015.

LI, Y.; ZHANG, L. The Behavior Modes of Multiple Agents in Knowledge Management System Based on Complexity Theory. In: INTERNATIONAL CONFERENCE ON INTELLIGENCE SCIENCE AND INFORMATION ENGINEERING, 2011, Wuhan [Conference Publications...], 2011. p. 53-56. Disponivel em: <http://dx.doi.org/10.1109/ISIE.2011.142>. Acesso em: 25 ago. 2015 MERRICK, T. R. The role of public libraries in knowledge cities. Library Student Journal, v. 4, $2009 . \quad$ Disponível em: $<$ http://search.ebscohost.com/login.aspx?direct=true\&db=lih\&AN=67426006\&lang=ptbr\&site=ehost-live $>$. Acesso em: 26 jul. 2015.

MONAGAS-DOCASAL, M. El capital intelectual y la gestión del conocimiento. Ingeniería Industrial, v. 32, n. 2, maio-ago. 2012.

NONAKA, I; TAKEUCHI, H. Criação de conhecimento na empresa. Rio de Janeiro: Campus, 1997.

PACHECO, A. P. R. et al. O ciclo PDCA na gestão do conhecimento: uma abordagem sistêmica. [2005?]. Disponível em: <http://www.isssbrasil.usp.br/isssbrasil/pdfs2/ana.pdf>. Acesso em: 26 jul. 2015.

PORUMBEANU, O. L. Strategic model for implementing knowledge management in libraries or information services. Library \& Information Science Research, n. 13, p. 89-105, 2009. Disponível em: $<$ http://search.ebscohost.com/login.aspx?direct=true\&db=lih\&AN=47272043\&lang=ptbr\&site=ehost-live>. Acesso em: 26 julho 2015.

SHUHUAl, R. et al. From information commons to knowledge commons: building a collaborative knowledge-sharing environment for innovative communities. The Electronic Library, v. 27, n. 2, p. 247 - 257, 2009. Disponível em: <http://dx.doi.org/10.1108/02640470910947593>. Acesso em: 26 jul. 2015.

UNIVERSIDADE FEDERAL DE SANTA CATARINA. Portaria no 849/2015/GR, de 25 de maio de 2015. Florianópolis, 2015a. 
UNIVERSIDADE FEDERAL DE SANTA CATARINA. Sistema de Bibliotecas Universitárias. Florianópolis, 2015. Disponível em: <http://portal.bu.ufsc.br/>. Acesso em: 14 ago. 2015b.

WORKSHOP GESTÃO DO CONHECIMENTO EM BIBLIOTECAS UNIVERSITÁRIAS, 1., 2014, Florianópolis. Relatório. Florianópolis: UFSC, 2014. Disponível em: <http://workshopgestaoconhecimentobu.paginas.ufsc.br>. Acesso em: 26 ago. 2015.

XIN QU, Y. Research of Knowledge Management on the University Library for Subject User. In: INTERNATIONAL CONFERENCE ON MANAGEMENT SCIENCE AND INDUSTRIAL ENGINEERING, 2011, Harbin. Anais... Washington: IEEE, 2011. p. 932-934. Disponível em: $<$ http://ieeexplore.ieee.org/stamp/stamp.jsp?tp=\&arnumber=5707563>. Acesso em: 26 ago. 2015.

Recebido/Recibido/Received: 2015-09-04 Aceitado/Aceptado/Accepted: 2015-10-08 
ANEXO 1 - Critérios de verificação para Reconhecimento da Biblioteca Universitária usando a visão dos Sistemas Adaptativos Complexos (SAC)

\begin{tabular}{|c|c|c|c|c|}
\hline $\begin{array}{l}\text { CRITÉRIOS DE VERIFICAÇÃO } \\
\text { (A Biblioteca Universitária...) }\end{array}$ & $\begin{array}{c}\text { Relação } \\
\text { com... }\end{array}$ & Nível & Prazo & Resp. \\
\hline $\begin{array}{l}\text { 1. Identifica e categoriza seus agentes em } \\
\text { tipologias, populações. As categorias } \\
\text { agrupam os agentes por similaridade. } \\
\text { Exemplos: } \\
\text { Agente usuário Agente funcionário } \\
\text { Agente fornecedor Agente Parceiro } \\
\text { Agente... }\end{array}$ & $\begin{array}{c}\text { Pessoas, } \\
\text { cultura e } \\
\text { estrutu- } \\
\text { ra }\end{array}$ & & & \\
\hline $\begin{array}{l}\text { 2. Conhece os principais agentes a serem } \\
\text { focados na BU. É preciso identificar os } \\
\text { agentes mais importantes, com maior } \\
\text { impacto sobre as atividades-fim da BU e } \\
\text { mais necessários para compreender a } \\
\text { complexidade do sistema, em seguida criar } \\
\text { categorias para esses agentes, para } \\
\text { futuramente entender como eles geram } \\
\text { variedade no seu comportamento, como } \\
\text { eles interagem uns com os outros, e como } \\
\text { os agentes e suas estratégias são } \\
\text { selecionados para conservação, expansão, } \\
\text { ou extinção. Exemplos: } \\
\text { Agente usuário Agente funcionário }\end{array}$ & $\begin{array}{c}\text { Conheci- } \\
\text { mento } \\
\text { interno }\end{array}$ & & & \\
\hline
\end{tabular}

(continua) 


\begin{tabular}{|c|c|c|c|c|}
\hline $\begin{array}{l}\text { CRITÉRIOS DE VERIFICAÇÃo } \\
\text { (A Biblioteca Universitária...) }\end{array}$ & $\begin{array}{c}\text { Relação } \\
\text { com... }\end{array}$ & Nível & Prazo & Resp. \\
\hline $\begin{array}{l}\text { 3. Identifica as principais estratégias, além de } \\
\text { ideias, regras, rotinas e normas utilizadas } \\
\text { pelos agentes ao agirem. Lembrando que } \\
\text { todos estes aspectos devem ser levantados } \\
\text { juntamente com a equipe, ou seja, os próprios } \\
\text { agentes. Exemplos: } \\
\text { coleções } \\
\text { *... Estratégia de atendimento }\end{array}$ & & & & \\
\hline $\begin{array}{l}\text { 4. Possui seus processos mapeados. Caso a BU } \\
\text { ainda não tenha o mapeamento de processos } \\
\text { (conjunto de atividades realizadas para atingir } \\
\text { um serviço) estabelecido de modo formal, será } \\
\text { necessário desenvolvê-lo. A partir do } \\
\text { mapeamento dos processos-chave da BU é } \\
\text { possível identificar, entre outros elementos, os } \\
\text { pontos fortes e fracos, os conhecimentos e } \\
\text { agentes envolvidos, o nível de complexidade } \\
\text { da operação. Exemplos de processos } \\
\text { organizacionais chave: } \\
\checkmark \quad \text { Desenvolver/atualizar a política de } \\
\quad \text { Desenvolvimento de Coleções; } \\
\checkmark \quad \text { Administrar Portal de Periódicos; } \\
\checkmark \quad \text { Realizar atendimento de Referência; } \\
\checkmark \quad \text { Fazer estudo de usuários; } \\
\checkmark \quad \text { Gerar relatórios estatísticos; } \\
\checkmark \quad \text { Promover aquisição (compra, permuta e } \\
\quad \text { doação); } \\
\checkmark \text { Realizar descarte e remanejamento; } \\
\checkmark \text { Efetuar empréstimos. }\end{array}$ & & & & \\
\hline
\end{tabular}




\begin{tabular}{|c|c|c|c|c|}
\hline $\begin{array}{l}\text { CRITÉRIOS DE VERIFICAÇÃO } \\
\text { (A Biblioteca Universitária...) }\end{array}$ & $\begin{array}{l}\text { Relação } \\
\text { com... }\end{array}$ & Nível & Prazo & Resp. \\
\hline $\begin{array}{l}\text { 5. Conhece as classificações que os próprios } \\
\text { agentes fazem para outros agentes em } \\
\text { populaç̃̃es e tipos. Algumas classificações são } \\
\text { necessárias para compreendermos que há } \\
\text { necessidades diferentes entre os agentes, no } \\
\text { caso das BU os usuários são uma boa } \\
\text { aplicação. Exemplos: } \\
\checkmark \quad \text { usuário inconveniente (aquele que exige } \\
\text { insistentemente da biblioteca uma } \\
\text { demanda que ela não oferece, como } \\
\text { estender horário de abertura para } \\
\text { finalidades específicas ou normalizar } \\
\text { trabalhos acadêmicos, por exemplo); } \\
\text { usuário calouro (demandas básicas como } \\
\text { informações de empréstimo, cadastro); } \\
\text { usuário de infraestrutura (utiliza a } \\
\text { biblioteca exclusivamente como espaço } \\
\text { físico); } \\
\text { usuário de elite (dúvidas pontuais e } \\
\text { difíceis de serem respondidas, exigindo } \\
\text { um profissional extremamente } \\
\text { qualificado e dispendendo bastante } \\
\text { tempo deste). }\end{array}$ & & & & \\
\hline
\end{tabular}




\begin{tabular}{|c|c|c|c|c|}
\hline $\begin{array}{l}\text { CRITÉRIOS DE VERIFICAÇÃO } \\
\text { (A Biblioteca Universitária...) }\end{array}$ & $\begin{array}{c}\text { Relação } \\
\text { com... }\end{array}$ & Nível & Prazo & Resp. \\
\hline $\begin{array}{l}\text { 6. Tem consciência dos artefatos, recursos e } \\
\text { ferramentas utilizados pelos agentes na } \\
\text { execução de suas estratégias. Exemplos: } \\
\text { Mobiliário (mesa, cadeira, estantes, } \\
\text { arquivos, } \\
\text { pastas, projetor etc.); } \\
\text { Artefatos de informação (livros, periódicos, } \\
\text { artigos, trabalhos acadêmicos etc.); } \\
\text { Ferramentas da internet e computacionais } \\
\text { (catálogos, bases de dados, gerenciadores } \\
\text { bibliográficos, metabuscadores, } \\
\text { ferramentas de descobrimento, softwares } \\
\text { variados); } \\
\text { Computadores e similares. } \\
\text { Recursos de compartilhamento de } \\
\text { conhecimento (catalogação, acervo etc.). }\end{array}$ & & & & \\
\hline $\begin{array}{l}\text { 7. Define os espaços físico, conceitual e digital } \\
\text { em que os agentes atuam. Exemplos: } \\
\checkmark \quad \text { Espaço físico: inserir informações como } \\
\text { metragem, plantas, localização, fotos etc.; } \\
\checkmark \quad \text { Espaço conceitual: inserir organograma, } \\
\text { comunidades de prática, grupos de } \\
\text { trabalho etc.; } \\
\checkmark \text { Espaço virtual/digital: páginas na } \\
\text { internet, portais, e-mail, chat, redes } \\
\text { sociais etc. }\end{array}$ & & & & \\
\hline
\end{tabular}

Fonte: Bem (2015, p. 209-211). 